Pre-Print Manuscript of Article:

Bridgelall, R., "Precision bounds of pavement deterioration forecasts from connected vehicles," Journal of Infrastructure Systems, American Society of Civil Engineering, 21(3), pp. 1-7, 2014.

\title{
Precision bounds of pavement deterioration forecasts from connected vehicles
}

5 Abstract

6 Transportation agencies rely on models to predict when pavements will deteriorate to a condition or ride-index threshold that triggers maintenance actions. The accuracy and precision

8 of such forecasts are directly proportional to the frequency of monitoring. Ride indices derived

9 from connected vehicle sensor data will enable transformational gains in both the accuracy and

10 precision of deterioration forecasts because of very high data volume and update rates. This

11 analysis develops theoretical precision bounds for a ride index called the road impact factor and

12 demonstrates, via a case study, its relationship with vehicle suspension parameter variances.

13 CE Database subject headings: Deterioration; Forecasting; Intelligent transportation systems;

14 Pavement management; Preservation; Probe instruments; Surface roughness; Vibration

15 Author Keywords: Connected vehicles; International Roughness Index; Ride quality; Road

16 Impact Factor

\section{Introduction}

18 Practitioners have long recognized that rough roads increase vehicle operating costs

19 (Zaniewski and Butler 1985) and lead to more expensive road repairs (AASHTO 2009). Studies

20 have also linked rough roads to motion sickness (Griffin 1990) and higher crash rates (Swedish

\footnotetext{
${ }^{1}$ Assistant Professor of Transportation and Program Director, Center for Surface Mobility Applications \& Real-Time Simulation environments (SMARTSe ${ }^{\mathrm{SM}}$ ), Upper Great Plains Transportation Institute, North Dakota State University, P.O. Box 863676, Plano, TX 75086. Phone: 408-607-3214, E-mail: raj@bridgelall.com

Raj Bridgelall, Ph.D. 
21 National Road and Transport Research Institute 2004). The ability to accurately predict

22 optimum maintenance cycles has the greatest potential impact on reducing annual maintenance

23 and rehabilitation costs (Madanat, Prozzi and Han 2002). More frequent condition assessments

24 increase the accuracy and precision of predicting when ride-quality indices will reach

25 maintenance thresholds (Haider, Baladi and Chatti 2011). Rural regions that maintain roads

26 suffering rapid deterioration caused by high levels of industrial and agricultural activities will

27 yield the greatest potential benefits from frequent condition assessments (Tolliver and Dybing

28 2012). Unfortunately, transportation agencies can seldom afford to assess ride-quality more

29 often than once a year. Even so, those assessments are limited to portions of the National

30 Highway System for which the Federal Highway Administration (FHWA) requires annual

31 reporting of the International Roughness Index (IRI) (HPMS 2012). Consequently, agencies

32 miss important vulnerabilities such as frost-heaves that appear and disappear between monitoring

33 cycles.

34 To provide continuous, network-wide, lower-cost ride-quality measures, the author developed

35 and validated a new approach called the Road Impact Factor (RIF). The average RIF collected

36 from inertial sensors onboard vehicles is directly proportional to the IRI (Bridgelall 2014).

37 Statistically, the RIF variance diminishes exponentially as the volume of sensor readings

38 increase. Using data from inertial sensors in smartphones and connected vehicles to produce the

39 RIF will provide highly precise and continuous ride-quality assessments.

40 This study characterizes the bounds in forecast precision for common regression models in

41 terms of RIF variability. The latter is a function of motion parameter distributions such as

42 vehicle suspension rates, ground speed, and traversal volume. This is the first study to relate

43 statistics of the RIF to the precision of deterioration forecasts. Related studies use the output of 
44 inertial sensors to estimate the IRI by calibrating the acceleration responses of individual

45 vehicles to known values (Nagayama, et al. 2013), or by estimating parameters of an IRI model

46 using neural networks and other methods (Dawkins, et al. 2011).

47 This paper is organized as follows: Section 2 briefly reviews the RIF model defined in

48 previous research. Section 3 relates the RIF to normalized vertical acceleration energy.

49 Section 4 links statistics of vehicle suspension parameters to the normalized vertical acceleration

50 energy and the RIF. Section 5 derives a model that relates the minimum traversal volume to a

51 level of forecast precision using a common regression model of pavement deterioration.

52 Section 6 presents a case study of the forecast precision bound for a typical distribution of

53 vehicle suspension parameters. Section 7 summarizes and concludes the study.

\section{$54 \quad 2$ Ride-Index Model}

55 As derived in previous work by the author, the RIF, denoted $R^{L}[p]$, is the g-force per meter $56(\mathrm{~g} / \mathrm{m})$ experienced when traveling a road segment of length $L$, during time-period $p$ where:

$$
R^{L}[p]=\sqrt{\frac{1}{L} \int_{0}^{L / \bar{\sigma}}\left|g_{z}(t) \sigma(t)\right|^{2} d t}
$$

57 The instantaneous traversal speed is $\sigma(t)$ and the on-board sensor output for vertical acceleration 58 is $g_{z}(t)$.

\section{Statistics of Vehicle Response Energy}

60 Road roughness excites the vibration modes of a moving vehicle. The damped mass-spring

61 model for each wheel-suspension assembly or "quarter-car" includes a series combination of

62 sprung and unsprung masses that represent a portion of the body and wheel components

63 respectively. A pair of second-order differential equations characterizes each model. Their 
64 solution identifies the dominant resonant frequencies and damping ratios of each mode as

65 functions of the vehicle body mass, wheel mass, spring stiffness, and damping coefficients.

66 These physical parameters must be known to determine the characteristics of each quarter-car

67 mode (Angeles 2011).

68 The vertical response $z(t)$ to a common broad-band input, namely an impulse, excites all

69 modes equally. The impulse responses of the $n$ under-damped mass-spring systems are:

$$
z_{[m, n]}(t)=\frac{U(t)}{\omega_{[m, n]} \sqrt{1-\zeta_{[m, n]}^{2}}} \exp \left(-\zeta_{[m, n]} \omega_{[m, n]} t\right) \sin \left(\omega_{[m, n]} \sqrt{1-\zeta_{[m, n]}^{2}} t\right)
$$

70 The subscripts $m=1$ and $m=2$ enumerate the sprung $(s)$ and unsprung $(u)$ mass-spring

71 subsystem parameters respectively. $U(t)$ is the Heaviside step function. The sprung and

72 unsprung mass resonance frequencies are $\omega_{[s, n]}$ and $\omega_{[u, n]}$ respectively, and their corresponding

73 damping ratios are $\zeta_{[s, n]}$ and $\zeta_{[u, n]}$. The Fourier Transform of the impulse response is a second-

74 order low-pass filter (LPF), $Z(\omega)$, of the form:

$$
Z_{[m, n]}(\omega)=\frac{1}{\sqrt{1-\zeta_{[m, n]}^{2}}} \frac{1}{\left(\omega_{[m, n]}\right)^{2}+\left(\zeta \omega_{[m, n]}+j \omega\right)^{2}}
$$

75 At the sensor's location, the vertical acceleration $G_{\alpha}$ is a product of the sensor frequency

76 response $S(f)$ and the vector sum of responses from each quarter-car. A linear combination of the

77 mass-spring models for each wheel-assembly produces an equivalent but more analytically

78 convenient model of the acceleration vector, $G_{\beta}$ as illustrated in Figure 1. Hence, the magnitude

79 spectrum of the composite vertical acceleration response is:

$$
\left|G_{\beta}(f)\right|=|S(f)| \sum_{n=1}^{W} \sum_{m=1}^{2} \beta_{[m, n]} \frac{1}{4 \pi^{2}} \frac{1}{\sqrt{1-\zeta_{[m, n]}^{2}}} \frac{1}{\left.\sqrt{\left(\left[f_{[m, n]}^{2}-f^{2}\right]^{2}+\left[2 \zeta_{[m, n]} f_{[m, n]} f\right]^{2}\right.}\right)}
$$


80 The frequencies in hertz are $f_{[m, n]}=\omega_{[m, n]} / 2 \pi . W$ is the number of wheel-spring assemblies and

$81 \beta_{[m, n]}$ are the coefficients of the linear combination. The LPF filter gains are:

$$
A_{[m, n]}=\frac{1}{4 \pi^{2} \sqrt{\left(1-\zeta_{[m, n]}^{2}\right)}}
$$

82 Solving for the sprung and unsprung mass filter coefficients $\beta_{[s, n]}$ and $\beta_{[u, n]}$ respectively such that

83 the energy of $G_{\beta}$ equals the energy of $G_{\alpha}$ yields:

$$
\beta_{[s, n]}=\frac{A_{[s, n]} A_{[u, n]}}{A_{[s, n]}+\rho_{[n]} A_{[u, n]}}=\frac{1}{4 \pi^{2}\left[\rho_{[n]} \sqrt{\left(1-\zeta_{[s, n]}^{2}\right)}+\sqrt{\left(1-\zeta_{[u, n]}^{2}\right)}\right]}
$$

84 and

$$
\beta_{[u, n]}=\frac{A_{[s, n]} A_{[u, n]}}{A_{[s, n]} 1 / \rho_{[n]}+A_{[u, n]}}=\frac{1}{4 \pi^{2}\left[\sqrt{\left(1-\zeta_{[s, n]}^{2}\right)}+1 / \rho_{[n]} \sqrt{\left(1-\zeta_{[u, n]}^{2}\right)}\right]}
$$

85 where the ratios:

$$
\rho_{[n]}=\frac{\beta_{[u, n]}}{\beta_{[s, n]}}
$$

86 depends on the vehicle design and sensor installation. Figure 2 plots the Discrete Fourier

87 Transform (DFT) of the vertical acceleration signal samples $\left\{g_{z}\right\}$ obtained from a passenger car

88 used in related studies. A least squares fit of the quarter-car model in Equation (4) with $\mathrm{W}=1$

89 provided a ratio of $\rho=2.4$. The sprung and unsprung mass resonant modes for each quarter-car

90 are observable near 1.5 and 11 hertz respectively. In related work pending publication by the

91 author, a ratio of $\rho=4.0$ was observable less than $5 \%$ of the time from hundreds of traversals of

92 the same road segment, using several types of passenger vehicles. 
93 The suspension parameters from vehicles traversing a road segment will result in a statistical

94 distribution of impulse responses with vertical acceleration energy:

$$
E_{[m, n]}=\int_{0}^{\infty}\left|g_{[m, n]}(t)\right|^{2} d t=\int_{0}^{\infty}\left|\frac{d^{2}}{d t^{2}} z_{[m, n]}(t)\right|^{2} d t=\omega_{[m, n]}\left(\zeta_{[m, n]}+\frac{1}{4 \zeta_{[m, n]}}\right)
$$

95 where $g_{[m, n]}$ are the vertical accelerations from the individual mass-spring impulse responses.

96 The vertical acceleration vector at the sensor's position is:

$g_{z}(t)=\sum_{n=1}^{W} \sum_{m=1}^{2} \beta_{[m, n]} g_{[m, n]}(t)$

97 Therefore, the corresponding acceleration energy $E_{g z}$ is:

$$
E_{g z}=\sum_{n=1}^{W} \sum_{m=1}^{2} \int_{0}^{\infty}\left|\beta_{[m, n]} g_{[m, n]}(t)\right|^{2} d t=\sum_{n=1}^{W} \sum_{m=1}^{2} \beta_{[m, n]}^{2} \omega_{[m, n]}\left(\zeta_{[m, n]}+\frac{1}{4 \zeta_{[m, n]}}\right)
$$

98 From the theory of error propagation $(\mathrm{Ku} 1966)$, the acceleration energy variance is:

$$
v E_{g z}=\sum_{n=1}^{W} \sum_{m=1}^{2}\left[\left(\frac{\partial E_{g z}}{\partial \omega_{[m, n]}}\right)^{2} s_{\omega[m, n]}^{2}+\left(\frac{\partial E_{g z}}{\partial \zeta_{[m, n]}}\right)^{2} s_{\zeta[m, n]}^{2}+\left(\frac{\partial E_{g z}}{\partial \omega_{[m, n]}}\right)\left(\frac{\partial E_{g z}}{\partial \zeta_{[m, n]}}\right) s_{\omega \zeta[m, n]}^{2}\right]
$$

99 where $s_{\omega[m, n]}^{2}, s_{\zeta[m, n]}^{2}$ and $s_{\omega \zeta[m, n]}^{2}$ are the variances of the mode resonant frequencies, damping

100 ratios, and their covariance factors respectively. The latter is zero because the resonant

101 frequencies and damping ratios are statistically independent. Substituting the partial derivatives

102 indicated yield:

$$
v E_{g z}=\sum_{n=1}^{W} \sum_{m=1}^{2}\left[\beta_{[m, n]}^{4}\left(\zeta_{\mu[m, n]}+\frac{1}{4 \zeta_{\mu[m, n]}}\right)^{2} s_{\omega[m, n]}^{2}+\left(\nabla E_{\zeta[m, n]}\right)^{2} s_{\zeta[m, n]}^{2}\right]
$$

103 where 


$$
\nabla E_{\zeta[s, n]}=\frac{2 \rho_{[n]}^{3} \omega_{u, n]} \zeta_{[s, n]}^{3}\left(4 \zeta_{[u, n]}^{2}+1\right)+\omega_{s, n]} \zeta_{[u, n]}\left\{\rho_{[n]}\left[\zeta_{[s, n]}^{2}\left(4 \zeta_{[s, n]}^{2}+7\right)-1\right]+\sqrt{\left(1-\zeta_{[s, n]}^{2}\right)\left(1-\zeta_{[u, n]}^{2}\right.}\left(4 \zeta_{[s, n]}^{2}+1\right)\right\}}{64 \pi^{2} \zeta_{[s, n]}^{2} \zeta_{[u, n]}\left\{\rho_{[n]} \sqrt{1-\zeta_{[s, n]}^{2}}+\sqrt{1-\zeta_{[u, n]}^{2}}\right\} \sqrt{1-\zeta_{[s, n]}^{2}}}
$$

104 and

$$
\nabla E_{\zeta[u, n]}=\frac{2 \omega_{s, n]} \zeta_{[u, n]}^{3}\left(4 \zeta_{[s, n]}^{2}+1\right)+\rho_{[n]}^{2} \omega_{l u, n]} \zeta_{[s, n]}\left\{\zeta_{[u, n]}^{2}\left(4 \zeta_{[u, n]}^{2}+7\right)+\rho_{[[n]}\left(4 \zeta_{[u, n]}^{2}-1\right) \sqrt{\left(1-\zeta_{[s, n]}^{2}\right)\left(1-\zeta_{[u, n]}^{2}\right)}-1\right\}}{64 \pi^{2} \zeta_{[s, n]} \zeta_{[u, n]}^{2}\left\{\rho_{[n]} \sqrt{1-\zeta_{[s, n]}^{2}}+\sqrt{1-\zeta_{[u, n]}^{2}}\right\} \sqrt[3]{1-\zeta_{[u, n]}^{2}}}
$$

105 This energy variance is an important factor of the RIF variance derived in the next section.

\section{RIF Variance}

108 produces a RIF of:

$$
R_{\bar{\sigma}}^{L}=\bar{\sigma}_{\kappa} \sqrt{\frac{1}{L} \int_{0}^{L / \bar{\sigma}}\left|g_{z}(t)\right|^{2} d t}=\bar{\sigma}_{\kappa} \sqrt{\frac{1}{L}} \sqrt{\lim _{\varepsilon \rightarrow 0} \int_{0}^{T_{\varepsilon}}\left|g_{z}(t)\right|^{2} d t}=\bar{\sigma}_{\kappa} \sqrt{\frac{1}{L}} \sqrt{E_{g z}}
$$

109 where $T_{\varepsilon}=\left|g_{z}^{-1}(\varepsilon)\right|$. That is, the limit of integration is when the vertical acceleration of the

110 impulse response vector becomes negligibly small. The standard deviation of the RIF, $s_{R I F}^{L}$ is,

111 therefore:

$$
s_{R I F}^{L}=\sqrt{\left(\frac{\partial R_{\bar{\sigma}}^{L}}{\partial \bar{\sigma}_{\kappa}}\right)^{2} v\left[\bar{\sigma}_{\kappa}\right]+\left(\frac{\partial R_{\bar{\sigma}}^{L}}{\partial E_{g z}}\right)^{2} v E_{g z}+\left(\frac{\partial R_{\bar{\sigma}}^{L}}{\partial \bar{\sigma}_{\kappa}}\right)\left(\frac{\partial R_{\bar{\sigma}}^{L}}{\partial E_{g z}}\right) s_{\overline{\sigma E}}^{2}}
$$

112 where $v\left[\bar{\sigma}_{\kappa}\right]$ is the variance of the mean speed among traversals. The covariance of the mean

113 speed and the vertical acceleration signal energy, denoted $s_{\overline{\sigma E}}^{2}$, is zero because of their statistical

114 independence. Evaluating the partial derivates indicated in Equation (17) yields: 


$$
s_{R I F}^{L}=\sqrt{\frac{1}{L}\left(\bar{E}_{g z} v\left[\bar{\sigma}_{\kappa}\right]+\frac{\left(\overline{\bar{\sigma}}_{\kappa} / 2\right)^{2}}{\bar{E}_{g z}} v E_{g z}\right)}
$$

115 where $\bar{E}_{g z}$ and $\overline{\bar{\sigma}}_{\kappa}$ are respectively the mean vertical acceleration signal energy and the mean of

116 the average speed among traversals. This expression establishes a variance boundary for the

117 independent parameter of any related regression model used to predict a future deterioration

118 threshold.

\section{Deterioration Forecasting Models}

120 The most common models of pavement deterioration are empirical regression of the IRI because

121 they provide the greatest practical value and abstract the complexity of the underlying

122 phenomena (Lu and Tolliver 2012). Research demonstrates that IRI over time follows the

123 exponential form (S. W. Haider, et al. 2010):

$$
\psi(t)=\psi_{0} \exp \left(\beta_{L} t\right)
$$

124 where $\psi_{0}$ and $\psi(t)$ are respectively the initial and expected ride-indices at time $t$, and $\beta_{L}$ is a

125 calibration parameter that best fits the historical ride-index measured for segment $L$. Therefore,

126 the expected time to reach a given index threshold $\psi_{\alpha}$ is:

$$
\hat{T}\left(\psi_{\alpha}\right)=\frac{1}{\beta_{L}} \ln \left(\frac{\psi_{\alpha}}{\psi_{0}}\right)
$$

127 The estimate uncertainty for a future time, $\mathrm{s}_{\mathrm{T} \psi}$, to reach the ride-index threshold is:

$$
s_{T \psi}=\sqrt{\left[\frac{\partial T\left(\psi_{\alpha}\right)}{\partial \psi_{\alpha}}\right]^{2} s_{\psi \alpha}^{2}}=\frac{1}{\beta_{L}} \frac{s_{\psi \alpha}}{\psi_{\alpha}}
$$


128 where $\mathrm{s}_{\psi \alpha}$ is a window of uncertainty about the future ride-index threshold. Using RIF as the

129 ride-index, the ratio $s_{\psi \alpha} / \psi_{\alpha}$ is bounded by the ratio of the RIF standard deviation to the mean RIF

130 of quarter-car impulse responses where:

$$
\frac{s_{\psi \alpha}}{\psi_{\alpha}}=\frac{s_{R I F}^{L}}{R_{\bar{\sigma}}^{L}}=\sqrt{\frac{v\left[\bar{\sigma}_{K}\right]}{\overline{\bar{\sigma}}_{k}^{2}}+\frac{1}{4} \frac{v E_{g z}}{\bar{E}_{g z}^{2}}}
$$

131 The time margin-of-error $\Delta \mathrm{T}_{\psi}$ is:

$$
\Delta T_{\psi}=\frac{q_{1-\alpha / 2} \times s_{T \psi}}{\sqrt{N_{v}}}=\frac{q_{1-\alpha / 2}}{\sqrt{N_{v}}} \frac{1}{\beta_{L}} \frac{s_{R I F}^{L}}{R_{\bar{\sigma}}^{L}}
$$

132 where $N_{v}$ is the traversal volume, and $q_{1-\alpha / 2}$ is the standard normal quantile for a (1- $\left.\alpha\right) \%$

133 confidence interval (Papoulis 1991). Therefore, the minimum traversal volume needed to

134 achieve a minimum desired precision (maximum $\Delta T_{\psi}$ ) of the estimated time when the pavement

135 will deteriorate to a future ride-index $\psi$ is:

$$
N_{v}\left(\Delta T_{\psi}\right)=\left(\frac{1}{\Delta T_{\psi}} \frac{q_{1-\alpha / 2}}{\beta_{L}}\right)^{2}\left(\frac{v\left[\bar{\sigma}_{\kappa}\right]}{\bar{\sigma}_{k}^{2}}+\frac{1}{4} \frac{v E_{g z}}{\bar{E}_{g z}^{2}}\right)
$$

136 Given a deterioration rate parameter $\beta_{L}$, the precision $\Delta T_{\psi}$ is bounded by the sum of the standard

137 deviation-to-mean value ratios of the traversal velocity and vertical acceleration signal energy

138 respectively. The latter is bounded by the variance of the impulse response energy relative to the

139 mean response energy for all the vehicles that traverse the monitored road segment.

\section{Case Study}

\section{$141 \quad 6.1 \quad$ Vehicle Suspension Statistics}

142 It is standard practice for vehicle manufacturers to attenuate the vertical motion between 4

143 and 8 hertz because vibration levels within that frequency range are the most harmful to humans 
144 (Griffin 1990). To achieve this, manufacturers distribute the sprung and unsprung masses so that 145 they account for $90 \%$ and $10 \%$ respectively of the gross vehicle weight (Gillespie 2004). The 146 average curb weight of vehicles increased steadily since 1985 and peaked in 2007 (Bastani,

147 Heywood and Hope 2012), but trends indicate that they will return to 1990 levels by 2015 . The 148 average gross mass, $m_{\mu G}$, for vehicles manufactured in 2007 was 2226 kilograms and the 149 standard deviation, $s_{m G}$, was 483.7 kilograms (Woodyard 2007). These yield the mean and 150 standard deviations of the quarter-car sprung and unsprung masses, $m_{s}$ and $m_{u}$ respectively.

151 Suspension system engineers also design the sprung mass resonant frequency between 0.9 and 1521.5 hertz for all vehicle types (General Motors 1987). If this is approximately the six-sigma 153 range for normally distributed sprung mass resonant frequencies, $\omega_{s}$, of vehicles that travel any 154 road segment, then the mean frequency and standard deviation are 1.2 and 0.1 hertz respectively. 155 Similarly, vehicle suspension shock absorbers produce sprung mass damping ratios, $\zeta_{\mathrm{s}}$, in the 156 range of 0.3 to 0.4 (Gillespie 2004). Hence the mean and standard deviation for a normal 157 distribution is 0.35 and 0.017 respectively.

158 A tire at its rated load will experience a deflection of approximately $25 \mathrm{~mm}$ (Gillespie 2004), 159 therefore, for four-wheeled vehicles, an estimate of the average unsprung mass spring stiffness, $160 k_{u}$, in units of $\mathrm{N} \cdot \mathrm{m}^{-1}$ is:

$k_{u}=\frac{\left(m_{\mu G} / 4\right) g}{0.025}$

161 where $g$ is the g-force constant of $9.8 \mathrm{~m} \cdot \mathrm{s}^{-2}$. The mean unsprung mass resonant frequency, $\omega_{u}$, is 162 therefore:

$\omega_{u}=\sqrt{\frac{k_{u}}{m_{u}}}$ 
163 where $m_{u}$ is the average unsprung mass. From the gross mass statistics above, the associated

164 average resonant frequency is approximately 10 hertz. Its standard deviation is:

$$
s_{a u}=\sqrt{\left(\frac{\partial \omega_{u}}{\partial k_{u}}\right)^{2} s_{k u}^{2}+\left(\frac{\partial \omega_{u}}{\partial m_{u}}\right)^{2} s_{m u}^{2}+\left(\frac{\partial \omega_{u}}{\partial k_{u}}\right)\left(\frac{\partial \omega_{u}}{\partial m_{u}}\right) s_{k m}^{2}}
$$

165 For this scenario, both $k_{u}$ and $m_{u}$ depend on the gross vehicle mass statistics, therefore, the

166 covariance factor is unity and the expression becomes:

$s_{\omega u}=\sqrt{\frac{1}{4 k_{u} m_{u}} s_{k u}^{2}+\frac{k_{u}}{4 m_{u}^{3}} s_{m u}^{2}-\frac{1}{4 m_{u}^{2}}}$

167 The average damping ratio for the unsprung mass is defined as:

$$
\zeta_{u}=\frac{c_{u}}{2 \sqrt{m_{u} k_{u}}}=\frac{c_{u}}{2 m_{u} \omega_{u}}
$$

168 The unsprung mass damping coefficient $c_{u}$ is typically $\eta=15 \%$ of the sprung mass damping

169 coefficient $c_{s}$ (Türkay and Akçay 2008). Therefore,

$$
\zeta_{u}=\frac{\eta c_{s}}{2 m_{u} \omega_{u}}=\frac{\eta\left(2 m_{s} \omega_{s} \zeta_{s}\right)}{2 m_{u} \omega_{u}}=\eta \zeta_{s} \frac{m_{s}}{m_{u}} \frac{\omega_{s}}{\omega_{u}}
$$

170 where $m_{s}, \omega_{s}$, and $\zeta_{s}$ are the means of the sprung masses, their resonant frequencies, and their

171 damping ratios respectively. Hence, the standard deviation of the unsprung mass damping ratio

172 is:

$$
s_{\zeta u}=\sqrt{\left(\frac{\partial \zeta_{u}}{\partial \zeta_{s}}\right)^{2} s_{\zeta s}^{2}+\left(\frac{\partial \zeta_{u}}{\partial \omega_{s}}\right)^{2} s_{a s}^{2}+\left(\frac{\partial \zeta_{u}}{\partial \omega_{u}}\right)^{2} s_{a u}^{2}+\left(\frac{\partial \zeta_{u}}{\partial m_{s}}\right)^{2} s_{m s}^{2}+\left(\frac{\partial \zeta_{u}}{\partial m_{u}}\right)^{2} s_{m u}^{2}+\Delta_{c v}}
$$

173 where the covariance term $\nabla_{\mathrm{cv}}$ is:

$$
\Delta_{c v}=\left(\frac{\partial \zeta_{u}}{\partial m_{s}}\right)\left(\frac{\partial \zeta_{u}}{\partial m_{u}}\right) s_{m s m u}^{2}+\left(\frac{\partial \zeta_{u}}{\partial m_{s}}\right)\left(\frac{\partial \zeta_{u}}{\partial \omega_{u}}\right) s_{m s \omega u}^{2}+\left(\frac{\partial \zeta_{u}}{\partial m_{u}}\right)\left(\frac{\partial \zeta_{u}}{\partial \omega_{u}}\right) s_{m u \propto u}^{2}
$$


174 For this scenario, the variables $m_{s}, m_{u}$, and $\omega_{u}$ are proportionally linked per the guidelines for

175 typical vehicle suspension designs; therefore, their respective covariance factors $s_{m s m u}, s_{m s \omega u}$, and

$176 S_{\text {тиюи }}$ are unity. Evaluating the partial derivatives indicated, and simplifying yields:

$$
\Delta_{c v}=\left(\eta^{2} \zeta_{s}^{2} \frac{m_{s}}{m_{u}} \frac{\omega_{s}^{2}}{\omega_{u}^{2}}\right)\left[\frac{m_{s}-m_{u}-\omega_{u}}{m_{u}^{2} \omega_{u}}\right]
$$

177 With these typical values of vehicle parameters, all of values for the mean and variance of the

178 quarter-car suspension parameters specified in Equations (11) and (13) are now known to

179 compute a value for the energy variance to mean ratio of Equation (18). Table 1 summarizes the

180 ratios of standard deviations to mean values for the sprung and unsprung mass parameters of this

181 case study. With these values, Equation (24) will produce the number of sensor readings needed

182 for a specified level of forecast precision and confidence when given the average traversal speed,

183 its standard deviation, and the historical rate of pavement deterioration. The next section

184 provides an example based on a typical scenario.

\subsection{Deterioration Forecasting Example}

186 As shown in Figure 2, estimating a single quarter-car response from the aggregate provides a

187 reasonable simplification to accommodate the quarter-car statistics from the case study. Figure 3

188 plots Equation (24), normalized to the number of data collection days required for a desired

189 maximum forecast precision within a 95\% confidence interval. This result is based on an

190 average travel speed of $24.6 \mathrm{~m} / \mathrm{s}$ (about $55 \mathrm{mph}$ ) within a $5 \%$ standard deviation. The number of

191 data collection days depend on the typical Annual Average Daily Traffic (AADT) volume

192 medians of 10,965 and 39,093 passenger cars per lane for rural and urban Interstate functional

193 classifications respectively (Hausman and Clarke 2012), and a scenario where only 20\% of the

194 vehicles are equipped with sensors. The result is also based on typical rural and urban interstate 
195 highway deterioration rates (Anastasopoulos, Mannering and Haddock 2009), which correspond 196 to $\beta_{L}$ values of 0.056 and 0.055 respectively. The plot for this scenario indicates that one week

197 of data collection will forecast RIF thresholds with a worst-case precision of two weeks for the 198 typical urban and rural Interstate. To maintain homoscedasticity, the maximum data collection

199 period selected should be less than the maximum time-period that the deterioration level is

200 relatively unchanged.

\section{Summary and Conclusions}

202 The ability to collect and process data from a large number of inertial sensors in a connected 203 vehicle environment will provide transformational gains in the precision and accuracy of

204 forecasting pavement deterioration forecasts. Fundamentally, the accuracy and precision of a 205 regression model's ability to predict pavement deterioration is directly proportional to the rate of 206 its recalibration with new ride-quality data. Statistical properties of the road impact factor (RIF), 207 a new ride-index introduced in previous work, inherently improves its forecast precision as data 208 volume increases, making it an ideal model for a connected vehicle environment. This analysis 209 provides theoretical insights that relate the statistics of vehicle motion parameters to bounds of 210 its forecast precision. The supporting case study used suspension parameter variances published

211 for vehicles manufactured in 2007. A scenario with $20 \%$ of the passenger cars traveling a typical

212 U.S. interstate highway at a common speed limit and producing RIF data was analyzed. Results

213 for this scenario indicate that the model will predict a future ride-index within a worst case

214 precision of two weeks from statistics of RIF data collected for about one week.

215 Future work will characterize pavement distress symptom location accuracy in terms of the

216 variability of vehicle suspension response durations, errors in geospatial position estimates, and 217 asynchronous accelerometer and GPS sample rates. 


\section{Acknowledgement}

219 This work is based on research supported by the United Stated Department of Transportation

220 (USDOT), Research and Innovative Technology Administration (RITA) under the Rural

221 Transportation Research Initiative.

\section{Notation}

223 The following symbols are used in this paper:

$224 A_{[m, n]} \quad=\quad$ low-pass filter amplitude for mass-spring model $n$;

$225 \quad c_{s} \quad=\quad$ average damping coefficient of the sprung mass response motion;

$226 c_{u} \quad=\quad$ average damping coefficient of the unsprung mass response motion;

$227 \quad=\quad$ frequency in hertz;

$228 f_{[s, n]} \quad=\quad$ sprung mass resonance mode frequency (hertz) of quarter-car $n$;

$229 f_{[u, n]}=$ unsprung mass resonance mode frequency (hertz) of quarter-car $n$;

$230 g_{z}(t)=\quad$ aggregate g-force output from the inertial sensor as a function of time $t$;

$231 g_{[u, n]}(t)=\quad$ g-force sensed as a function of time $t$ from individual mass-spring models;

$232 \quad k_{u} \quad=\quad$ unsprung mass spring stiffness;

$233 L \quad=\quad$ length of road segment;

$234 m_{s} \quad=\quad$ the average sprung mass of vehicles;

$235 m_{u} \quad=\quad$ the average unsprung mass of vehicles;

$236 m_{\mu G} \quad=\quad$ average gross mass for vehicles;

$237 \quad N_{v} \quad=\quad$ the traversal volume;

$238 R^{L}[p] \quad=\quad$ RIF for segment of length $L$ evaluated in time-period $p ;$

$239 \quad R_{\bar{\sigma}}^{L} \quad=\quad$ RIF for segment of length $\mathrm{L}$ when traversed at an average speed $\bar{\sigma}$;

$240 \quad S_{k s}=\quad$ standard deviation of the sprung mass spring stiffness; 
$=\quad$ standard deviation of the unsprung mass spring stiffness;

$242 s_{k m}^{2}$

$243 S_{m s}$

$244 S_{m u}$

$245 s_{m s m u}$

$246 s_{m s \omega u}$

$247 s_{\text {mиюи }}$

$248 s_{R I F}^{L}$

$249 s_{T \psi}$

$250 s_{\omega s}$

$251 s_{\text {ои }}$

$252 S_{\psi \alpha}$

$253 S_{\zeta s}$

$254 S_{\zeta u}$

$255 s_{\overline{\sigma E}}^{2}$

$256 s_{\omega[m, n]}^{2}$

$257 s_{\omega \zeta[m, n]}^{2} \quad=$

$258 s_{\zeta[m, n]}^{2}$

$259 \quad S(f)$

$260 T_{\varepsilon}$

261

$q_{1-\alpha / 2}$
$=$

$=$

$=$

$=$

$=$

$=$

$=$

$=$

$=$

$=$

$=$

$=$

$=$

$=$

$=$

$=$

covariance of the unsprung mass and its spring stiffness;

standard deviation of the sprung mass;

standard deviation of the unsprung mass;

covariance between the sprung mass and the unsprung mass;

covariance between the sprung mass and unsprung mass resonance;

covariance between the unsprung mass and the unsprung mass resonance;

standard deviation of the RIF for segment of length $L$;

uncertainty of estimating the future time to reach a ride-index threshold $\psi$;

standard deviation of the sprung mass resonant frequency;

standard deviation of the unsprung mass resonant frequency;

the uncertainty band about a future ride-index threshold $\psi \alpha$;

standard deviation of the sprung mass damping ratio;

standard deviation of the unsprung mass damping ratio;

covariance of the average speed and vertical acceleration signal energy;

variances of the mode resonant frequencies;

covariances of the mode resonant frequencies and damping ratios;

the standard normal quantile for a $(1-\alpha) \%$ confidence interval;

Raj Bridgelall, Ph.D.

Page 15/22 
$262 v E_{g z} \quad=\quad$ the variance of acceleration energy;

$263 v\left[\bar{\sigma}_{\kappa}\right]=$ the variance of the mean speed among $\kappa$ traversals;

$264 \mathrm{~W} \quad=\quad$ the number of vehicle wheel-spring assemblies;

$265 U(t)=$ the Heaviside step function;

$266 z_{[m, n]}(t) \quad=\quad$ vertical motion of each mass-spring model;

$267 Z_{[m, n]}(\omega) \quad=\quad$ Fourier transform of the vertical motion of each mass-spring model;

$268 \beta_{[u, n]} \quad=\quad$ proportion of each LPF in the linear combination aggregate model;

$269 \beta_{L} \quad=\quad$ a calibration parameter that best fits the segment $L$ ride-index time series;

$270 \nabla_{\mathrm{cv}} \quad=\quad$ expression of the covariance factors among parameters;

$271 \Delta \mathrm{T}_{\psi} \quad=\quad$ the time margin-of-error relative to the ride-index standard deviation;

$272 \rho_{[u, n]} \quad=\quad$ ratio of unsprung to sprung mass quarter-car model $n$ coefficient;

$273 \quad \bar{\sigma}_{\kappa} \quad=\quad$ average or constant speed for the $\kappa^{\text {th }}$ traversal;

$274 \overline{\bar{\sigma}}_{\kappa} \quad=\quad$ mean of the average or constant speed among traversals;

$275 \sigma(t)=$ instantaneous traversal speed as a function of time;

$276 \omega_{s} \quad=\quad$ average sprung mass angular resonance frequency;

$277 \omega_{u} \quad=\quad$ average unsprung mass angular resonance frequency;

$278 \omega_{[s, n]} \quad=\quad$ sprung mass resonance mode angular frequency of quarter-car $n$;

$279 \omega_{[u, n]} \quad=\quad$ unsprung mass resonance mode angular frequency of quarter-car $n$;

$280 \psi_{0} \quad=\quad$ the initial ride-index at time $t=0$;

$281 \psi_{\alpha}=$ a ride-index threshold that triggers maintenance action;

$282 \psi(t)=$ the expected ride-index at time $t$;

$283 \zeta_{s} \quad=\quad$ average damping ratio of the sprung sprung mass frequency response;

Raj Bridgelall, Ph.D.

Page 16/22 
$284 \zeta_{u} \quad=\quad$ average damping ratio of the unsprung sprung mass frequency response;

$285 \zeta_{[s, n]} \quad=\quad$ damping ratios of the sprung mass frequency response;

$286 \zeta_{[u, n]} \quad=\quad$ damping ratios of the unsprung mass frequency response.

288 References

289 AASHTO. Rough Roads Ahead: Fix Them Now or Pay for It Later. Washington, DC: American 290 Association of State Highway and Transportation Officials (AASHTO) and The Road 291 Information Program (TRIP), 2009.

292 Anastasopoulos, Panagiotis Ch., Fred L. Mannering, and John Haddock. Effectiveness and 293 Service Lives/Survival Curves of Various Pavement Rehabilitation Treatments. Publication 294 FHWA/IN/JTRP-2008/12. Joint Transportation Research Program, Indiana: Indiana 295 Department of Transportation and Purdue University, 2009.

296 Angeles, Jorge. Dynamic Response of Lineaer Mechanical Systems: Modeling, Analysis and 297 Simulation. New York: Springer, 2011.

298 Bastani, Parisa, John B. Heywood, and Chris Hope. U.S. CAFE Standards -- Potential for 299 Meeting Light-duty Vehicle Fuel Economy Targets, 2016-2025. MIT Energy Initiative, 300 Boston, Massachusetts: Massachusetts Institute of Technology, 2012.

301 Bridgelall, Raj. "Connected Vehicle Approach for Pavement Roughness Evaluation." Journal of 302 Infrastructure Systems (American Society of Civil Engineers) 20, no. 1 (April 2014): $303 \quad 04013001$.

304 Dawkins, Jeremy, David Bevly, Buzz Powell, and Richard Bishop. Investigation of Pavement 305 Maintenance Applications of Intellidrive. Pooled Fund Study, Charlottesville, Virginia: 306 University of Virginia, 2011. 
General Motors. Suspension Rates and Frequency. Suspension Design Guidelines 1070, Detroit, 308 Michigan: General Motors, 1987.

309 Gillespie, Thomas D. CarSim Data Manual. Ann Arbor, Michigan: Mechanical Simulation $310 \quad$ Corporation, 2004.

311 Griffin, M. J. Handbook of Human Vibration. New York: Elsevier, 1990.

312 Haider, Sayed Waqar, Gilbert Y. Baladi, Karim Chatti, and Christopher M. Dean. "Effect of 313 Pavement Condition Data Collection Frequency on Performance Prediction." Transportation 314 Research Record: Journal of the Transportation Research Board (Transportation Research 315 Board), no. 2153 (2010): 67-80.

316 Haider, Syed Waqar, Gilbert Y. Baladi, and Karim Chatti. "Impact of Pavement Surface 317 Monitoring Frequency on Pavement Management Decision Making." Edited by Imad Al-Qadi 318 and Scott Murrell. Transportation and Development Institute Congress 2011. Chicago, 319 Illinois: American Society of Civil Engineers, 2011. 656-666.

320 Hausman, Joseph, and Justin Clarke. FHWA Functional Classification Guidance Update. Report, 321 Federal Highway Administration, Washington, DC: U.S. Department of Transportation, 2012. 322 HPMS. Highway Performance Monitoring System Field Manual (HPMS), Office of Highway 323 Policy Information Federal Highway Administration, Washington, DC: Federal Highway 324 Administration, 2012.

$325 \mathrm{Ku}$, Harry H. "Notes on the Use of Propagation of Error Formulas." Journal of Research of the 326 National Bureau of Standards, Section C: Engineering and Instrumentation (National Bureau 327 of Standards) 70C, no. 4 (1966): 263-273. 
Lu, Pan, and Denver Tolliver. "Pavement Pre- and Post-Treatment Performance Models Using LTPP Data." Journal of the Transportation Research Forum (Transportation Research

330 Forum) 51, no. 3 (2012): 67-81.

331 Madanat, Samer, Jorge A. Prozzi, and Michael Han. "Effect of Performance Model Accuracy on 332 Optimal Pavement Design." Computer-Aided Civil and Infrastructure Engineering 17, no. 1 333 (January 2002): 22-30.

334 Nagayama, Tomonori, Akira Miyajima, Shunya Kimura, Yuuki Shimada, and Yozo Fujino.

335 "Road condition evaluation using the vibration response of ordinary vehicles and 336 synchronously recorded movies." Proceedings of the SPIE 8692, Sensors and Smart 337 Structures Technologies for Civil, Mechanical, and Aerospace Systems 2013. Bellingham, 338 Washington: International Society for Optics and Photonics (SPIE), 2013.

339 Papoulis, Athanasios. Probalility, Random Variables, and Stochastic Processes. New York: $340 \quad$ McGraw-Hill, 1991.

341 Swedish National Road and Transport Research Institute. "The influence of road surface 342 condition on traffic safety and ride comfort." Sixth International Conference on Managing 343 Pavements: The Lessons, The Challenges, The Way Ahead. Brisbane, Queensland, Australia: 344 Queensland Department of Main Roads, 2004.

345 Tolliver, Denver, and Alan Dybing. An Assessment of County and Local Road Infrastructure 346 Needs in North Dakota. Research Report, Upper Great Plains Transportation Institute 347 (UGPTI), Fargo: North Dakota State University (NDSU), 2012.

348 Türkay, Semiha, and Hüseyin Akçay. "Influence of tire damping on the ride performance 349 potential of quarter-car active suspensions." 47th IEEE Conference on Decision and Control. 350 New York, New York: Institute of Electrical and Electronic Engineers (IEEE), 2008. 
351 Woodyard, Chris. Vehicles keep inching up and putting on pounds. July 16, 2007.

352 http://usatoday30.usatoday.com/money/autos/2007-07-15-little-big-cars_N.htm (accessed

353 March 14, 2014).

354 Zaniewski, John P., and Bert C. Butler. "Vehicle Operating Costs Related to Operating Mode, 355 Road Design, and Pavement Condition." In Measuring Road Roughness and Its Effects on

356 User Cost and Comfort, edited by Thomas D. Gillespie and Michael Sayer, 127-142.

357 Philadelphia: American Society for Testing and Materials, 1985.

358

359 


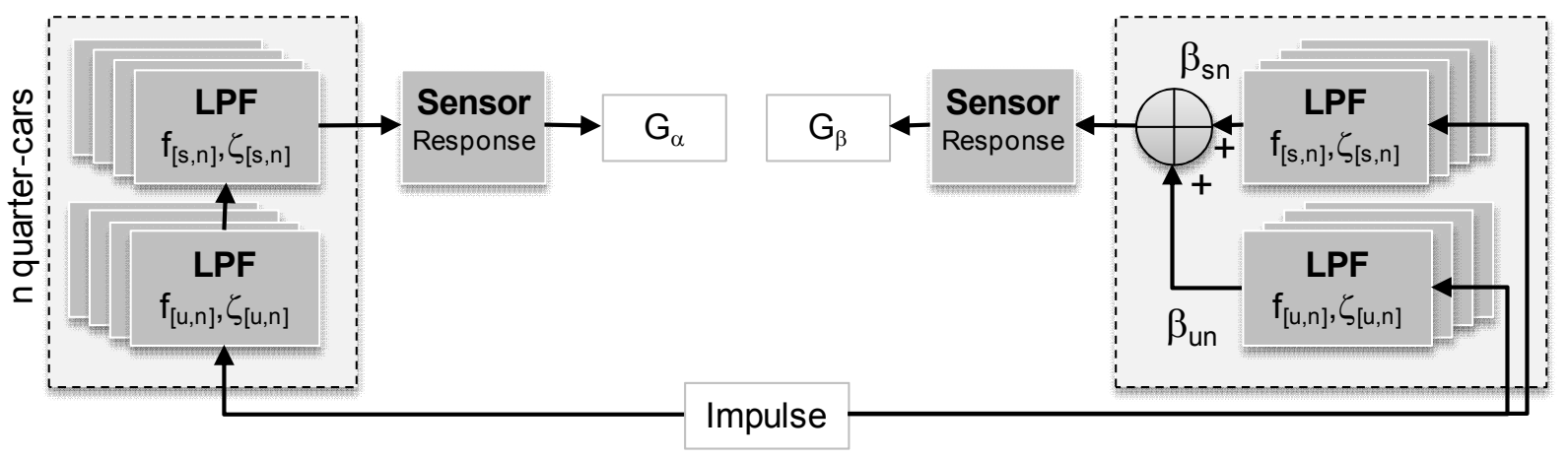

Figure 1. Equivalent response models of vehicle dynamics

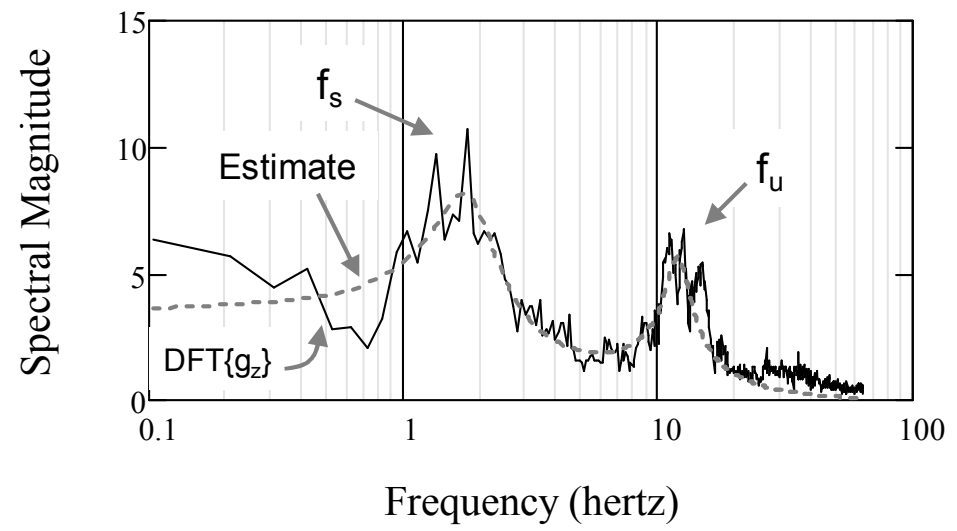

Figure 2. DFT of sensor output versus estimate of the quarter-car response 


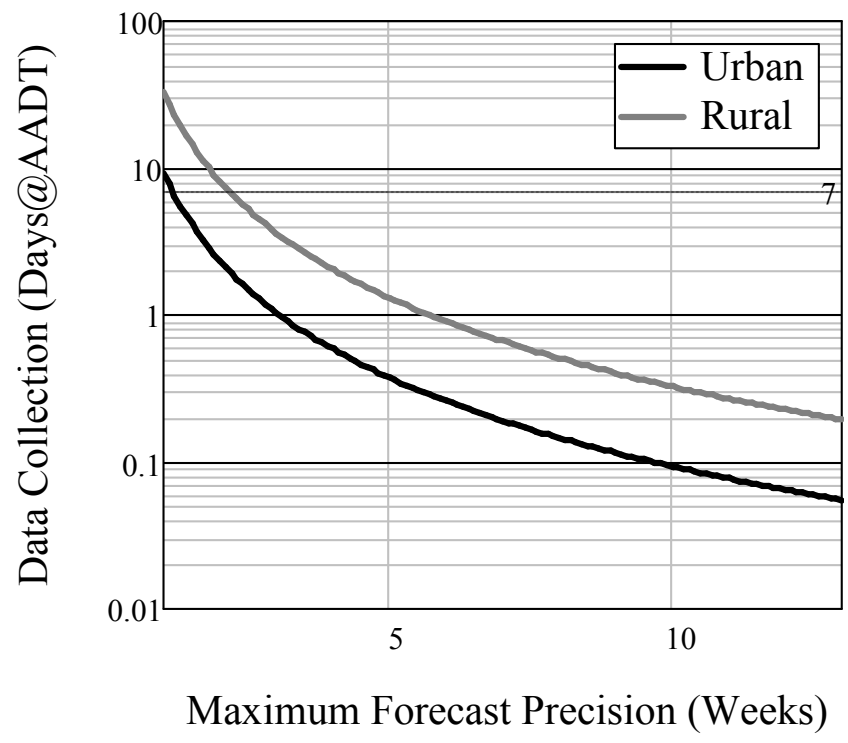

Figure 3. Data collection time needed for a desired maximum forecasting precision

Table 1: Ratio of standard deviation to the mean value for typical vehicles

\begin{tabular}{lcc}
\hline Parameter & Sprung Mass & Unsprung Mass \\
\hline Resonant Frequency $(\omega)$ & $8.3 \%$ & $15.4 \%$ \\
Damping Ratio $(\zeta)$ & $4.8 \%$ & $35.7 \%$ \\
Spring Stiffness $(k)$ & $27.4 \%$ & $21.7 \%$ \\
Damping Coefficient $(c)$ & $18.1 \%$ & $18.1 \%$ \\
\hline
\end{tabular}

